\title{
Stationary rotary force waves on the liquid-air core interface of a swirl atomizer
}

\author{
J. J. Chinn ${ }^{1}$ D. Cooper ${ }^{1}$ A. J. Yule ${ }^{2}$ G. G. Nasr ${ }^{3}$
}

Received: 6 January 2015 / Accepted: 25 October 2015 / Published online: 25 November 2015

(C) The Author(s) 2015. This article is published with open access at Springerlink.com

\begin{abstract}
A one-dimensional wave equation, applicable to the waves on the surface of the air-core of a swirl atomizer is derived analytically, by analogy to the similar one-dimensional wave equation derivation for shallowwater gravity waves. In addition an analogy to the flow of water over a weir is used to produce an analytical derivation of the flow over the lip of the outlet of a swirl atomizer using the principle of maximum flow. The principle of maximum flow is substantiated by reference to continuity of the discharge in the direction of streaming. For shallowwater gravity waves, the phase velocity is the same expression as for the critical velocity over the weir. Similarly, in the present work, the wave phase velocity on the surface of the air-core is shown to be the same expression as for the critical velocity for the flow at the outlet. In addition, this wave phase velocity is shown to be the square root of the product of the radial acceleration and the liquid thickness, as analogous with the wave phase velocity for shallow water gravity waves, which is the square root of the product of the acceleration due to gravity and the water depth. The work revisits the weirs and flumes work of Binnie et al. but using a different methodology. The results corroborate with the work of Binnie. High speed video, Laser
\end{abstract}

Electronic supplementary material The online version of this article (doi:10.1007/s00231-015-1706-0) contains supplementary material, which is available to authorized users.

\section{J. J. Chinn}

john.chinn@alumni.manchester.ac.uk

1 Faculty of Engineering and Physical Sciences, University of Manchester, Manchester, UK

2 CPF Research and Perdac, Sheffield, UK

3 Sprays Research Group, University of Salford, Manchester, UK
Doppler Anemometry and deflected laser beam experimental work has been carried out on an oversize Perspex (Plexiglas) swirl atomizer. Three distinctive types of waves were detected: helical striations, low amplitude random ripples and low frequency stationary waves. It is the latter wave type that is considered further in this article. The experimentally observed waves appear to be stationary upon the axially moving flow. The mathematical analysis allows for the possibility of a negative value for the phase velocity expression. Therefore the critical velocity and the wave phase velocity do indeed lead to stationary waves in the atomizer. A quantitative comparison between the analytically derived wave phase velocity and that measured experimentally, for this stationary pulsating wave, show very good agreement within a few percent.

\section{List of symbols}

$\mathrm{x}, \mathrm{r}, \theta \quad$ Cylindrical coordinate system

$\mathrm{u}, \mathrm{v}, \mathrm{w}$ Corresponding axial, radial and tangential velocity components

$\mathrm{u}_{\mathrm{c}} \quad$ Critical velocity at the constriction or throat

V Overall velocity

$\mathrm{r}_{\mathrm{ac}} \quad$ Air-core radius

$\mathrm{r}_{\mathrm{w}} \quad$ Wall radius

$r_{s} \quad$ Swirl chamber radius

$\mathrm{t}$ Time

S Surface boundary condition

$\mathrm{c}=\mathrm{wr}$ Free-vortex constant

$\mathrm{p} \quad$ Pressure

$\Delta \mathrm{p} \quad$ Pressure differential

$\varepsilon \quad$ Asymptotic power series parameter

$\eta \quad$ Wave height

$\rho_{\mathrm{L}} \quad$ Liquid density

$\chi \quad$ Wave phase velocity

Q Volumetric flow rate 
M Mass flow rate

h Water depth over a weir

g Gravitational field strength

\section{Introduction}

The phenomena of waves on liquid sheets issuing from atomizers, under relatively slow operating conditions, has been known for some time, Crapper et al. [1]. In particular, waves are the precursor to ligament and drop formation on the conical liquid sheets of swirl atomizers. For example, Nonnenmacher and Piesche [2] perform an analysis of aerodynamic wave break-up of a swirling liquid sheet. We postulate that the sheet waves originate from further upstream, on the surface of the air-core within the body of the atomizer.

\subsection{Centrifugal waves on the liquid air-core interface}

Crapper [3] and Stoker [4] both provide a mathematical derivation of a wave equation for long shallow water gravity waves. The work in this article is an analogical development for, what might be termed, rotary force waves, or centrifugal force waves, occurring on the air-core of a swirl atomizer. By substituting a swirling, rotating force acceleration for gravity it is shown that a wave equation applicable to the waves occurring on the surface of the air-core within swirl atomizers may be formulated.

\subsection{Critical velocity at the outlet}

In weir flow the surface topology and dynamics are governed by the volumetric flow rate and the force of gravity. In swirl atomizer flow the surface form and dynamics are governed by the volumetric flow rate and the analogous rotary, centrifugal, force. Following Taylors lead [5], several workers have attempted to determine the air-core diameter within a swirl atomizer by simple mathematical, analytical means: Giffen and Muraszew [6]., Bayvel and Orzechowski [7], Nieuwkamp [8] and Yule and Chinn [9]. A review of a number of these works, for these inviscid flows, is given in Chinn [10]. Once the air-core diameter in the outlet region was determined it was then a simple matter to calculate the axial velocity, the swirl velocity and the discharge coefficient (a measure of the volumetric flow rate for a given liquid density, operating pressure and outlet orifice diameter). It was then possible to make an estimate of the spray cone angle, and possibly even droplet size distribution by empirical means, Lefebvre [11].

For instance, Giffen and Muraszew [6] formulated an equation involving both the discharge coefficient and the air-core diameter in the outlet as unknowns. Having one equation and two unknowns is an unsolvable mathematical system. In order to alleviate this problem they invoke the 'principle of maximum flow'. In essence this means that the air-core diameter will adjust itself so that the volumetric flow rate will be a maximum. Following the usual method of determining a maximum or a minimum, the equation is rearranged so that the discharge coefficient is a function of the air-core. This is then differentiated and set equal to zero. One then obtains one equation in one unknown; the air-core diameter in the outlet orifice. Although the not-unreasonable assumption is made that the air-core will adjust itself so that the flow rate Q is a maximum $\left(\partial \mathrm{Q} / \partial \mathrm{r}_{\mathrm{ac}}=0\right)$, it is not substantiated. Binnie [12] asserts, in his work on flumes, that the component of gradient of mass flow in the direction of streaming, $\partial \mathrm{M} / \partial \mathrm{x}=0(\partial \mathrm{Q} / \partial \mathrm{x}=0$, for incompressible flow $)$ at every cross-section. This must be so for continuity, and is therefore a more robust assumption than simply assuming that the air-core size will adjust optimally. Binnie and Hooking [13] also worked on whirlpools through a trumpet mouth. The current article looks at both $[12,13]$. In this respect a constant pressure head, $\Delta \mathrm{p}$ is assumed to provide the flow motivation and gravity head and surface tension effects are considered negligible.

In the current article the principle of maximum flow for a swirl atomizer is formulated and it is shown that the film thickness in the orifice and the flow velocity, do indeed, adjust so that the flow rate is a maximum, and this is based on the continuity, $\partial \mathrm{Q} / \partial \mathrm{x}=0$.

The work provided here is based on three previous conference papers, with further additional work, enhancements and clarifications. The comparison of weir flow under gravity with that of flow over the lip of the outlet of a swirl atomizer, under centrifugal force, was first presented in [14]. The comparison of shallow water gravity waves with those on the surface of the air core under centrifugal force was first presented in [15]. A part of the experimental work, presented here, was first given in [16]. The proof of the validity of the critical flow velocity was first presented in [10], but without the wave phase velocity comparison, given in Sect. 2 below, and without the direct phase velocity comparison given in Sect. 4 below. The additions of the experimental work include a report on flow bifurcation and more detail of the experimental process. Comparison is made between some of the experimental results and the analytic theory.

The electronic supplementary material file (ESF), accompanying the present article, details both weir flow and the principle of maximum flow, first presented in [14], and shallow water gravity wave flow, first presented in [15]. These are provided for reference and are so configured to be directly analogous to the swirling flow work presented here. 


\subsection{Experimental work}

The results of imaging of the waves on the liquid interface of the air core of a swirl atomizer are presented. Three techniques were used: high speed video, Laser Doppler Anemometry (LDA) and deflected laser beam. The measurements show that the effects of the inlet ports are carried through to the spray cone and, it is theorised, contribute to the break-up of the spray. The axial (u) and tangential (w) velocity components in the swirl chamber were measured by means of LDA and the presence of localised recirculation regions in the liquid near to the air core were deduced. Stationary waves were observed on the air core in the swirl chamber. These were corroborated by LDA measurements of the axial and radial velocity components. Waves were also observed on the conical sheet issuing from the outlet using high speed imaging. The frequency of these waves corresponded to that of the stationary waves in the swirl chamber and it is theorised were caused by them. Internal unsteadiness of the liquid annulus within the outlet was observed.

\section{Long rotary-force waves on the air-core of a swirling flow through a nozzle}

This analysis was conducted in the cylindrical coordinate system $(\mathrm{x}, \mathrm{r}, \theta)$ with velocity components $(\mathrm{u}, \mathrm{v}, \mathrm{w})$. It is analogous to the analysis for shallow-water gravity waves in the Cartesian coordinate system (see ESF). These analyses are based on the Bernoulli equation for inviscid flows. An irrotational, in the mathematical sense, free-vortex flow is assumed. The flow of fluid was deemed to be axially symmetric so that terms differentiated w.r.t. $\theta$ are zero. The radial velocity $\mathrm{v}$, of the bulk flow, has been deemed to be zero in both the swirl chamber and the outlet orifice as the orifice wall provides an effective barrier to such movement. On the surface of the air-core there must clearly exist a local radial velocity normal to the mean free surface, in order to provide a wave amplitude, and so in this treatment the radial velocity has been retained. To facilitate the analysis the wave amplitude $\eta$ is considered to be of the form of an asymptotic power series in a small parameter $\varepsilon$, which is representative of the maximum wave slope, Stoker [4]. Again to facilitate the analysis, and without loss of generality the velocity $\mathbf{V}$ may also be considered to be of the form of an asymptotic power series in the small parameter $\varepsilon$.

$\eta(\mathrm{x}, \mathrm{t})=\varepsilon \eta_{0}+\varepsilon^{2} \eta_{1}+\varepsilon^{3} \eta_{2}+\cdots$

and

$\mathbf{V}=\varepsilon \mathbf{V}_{0}+\varepsilon^{2} \mathbf{V}_{1}+\varepsilon^{3} \mathbf{V}_{2}+\cdots$
At the fixed surface (wall) boundary, $r=r_{w}$, a surface function boundary condition, $S(r, x)=r_{w}-r=0$, may be employed. Similarly, at the free surface boundary, $r=r_{a c}-\eta$, a surface function boundary condition will be $S(r, x, t)=\left(r_{a c}-\eta\right)-r=0$. As the free surface boundary condition is $S=0$ then the total derivative of $S$ will also be zero, i.e. there is no temporal or spatial variation of this condition. The total, or full, derivative, in cylindricals, is

$\frac{\mathrm{DS}}{\mathrm{Dt}}=\frac{\partial \mathrm{S}}{\partial \mathrm{t}}+\underline{\mathrm{V}} \cdot \nabla \mathrm{S}=\frac{\partial \mathrm{S}}{\partial \mathrm{t}}+\mathrm{v} \frac{\partial \mathrm{S}}{\partial \mathrm{r}}+\frac{\mathrm{w}}{\mathrm{r}} \frac{\partial \mathrm{S}}{\partial \theta}+\mathrm{u} \frac{\partial \mathrm{S}}{\partial \mathrm{x}}$.

As an axisymmetric flow is being assumed then this can be written, for the free surface, as

$$
\begin{aligned}
\frac{\mathrm{DS}}{\mathrm{Dt}}= & \frac{\partial \mathrm{S}}{\partial \mathrm{t}}+\mathrm{v} \frac{\partial \mathrm{S}}{\partial \mathrm{r}}+u \frac{\partial \mathrm{S}}{\partial \mathrm{x}}=\frac{\partial}{\partial \mathrm{t}}\left[\left(\mathrm{r}_{\mathrm{ac}}-\eta\right)-\mathrm{r}\right] \\
& +\mathrm{v} \frac{\partial}{\partial \mathrm{r}}\left[\left(\mathrm{r}_{\mathrm{ac}}-\eta\right)-\mathrm{r}\right]+\mathrm{u} \frac{\partial}{\partial \mathrm{x}}\left[\left(\mathrm{r}_{\mathrm{ac}}-\eta\right)-r\right]=0 .
\end{aligned}
$$

Equation (4) may be simplified on the understanding of which parameters are not functions of a particular variable, also $r_{a c}$ is not a function of time and products of $v$ and $\eta$ are second order in $\varepsilon$, and hence negligible. Equation (4) may then be rearranged to form

$\frac{\partial \eta}{\partial \mathrm{t}}-\mathrm{u} \frac{\partial}{\partial \mathrm{x}}\left(\mathrm{r}_{\mathrm{ac}}-\eta\right)=-\mathrm{v}$.

The term $u \partial \mathrm{rac}_{\mathrm{ac}} / \partial \mathrm{x}$ is small compared to $\partial \eta / \partial \mathrm{t}$ and the term $\mathrm{u} \partial \eta / \partial \mathrm{x}$ is second order in the small parameter $\varepsilon$, and hence also small. On neglecting these terms Eq. (5) may be further reduced to

$\frac{\partial \eta}{\partial \mathrm{t}}=-\mathrm{v}_{\left(\mathrm{r}=\mathrm{r}_{\mathrm{ac}}-\eta\right)}$

where the subscript, $r=r_{a c}-\eta$, has been added to $\mathrm{v}$ in order to denote that this is the condition on the free surface.

The continuity equation may be written in cylindrical coordinates, with axial symmetry, as

$\nabla \cdot \underline{\mathrm{V}}=\frac{1}{\mathrm{r}} \frac{\partial}{\partial \mathrm{r}}(\mathrm{rv})+\frac{\partial \mathrm{u}}{\partial \mathrm{x}}=0$.

This may be rearranged and integrated between the limits of the top and bottom surfaces of the annulus of liquid in the body of the swirl atomizer, to form

$\int_{r=r_{W}}^{r=r_{a c}-\eta} \frac{\partial(r v)}{\partial r} d r=-\frac{\partial u}{\partial r} \int_{r=r_{w}}^{r=r_{a c}-\eta} r d r$,

as $\partial \mathrm{u} / \partial \mathrm{x}$ is not a function of $\mathrm{r}$. Thus

$\mathrm{r}_{\mathrm{w}} \mathrm{v}_{\left(\mathrm{r}=\mathrm{r}_{\mathrm{w}}\right)}-\left(\mathrm{r}_{\mathrm{ac}}-\eta\right) \mathrm{v}_{\left(\mathrm{r}=\mathrm{r}_{\mathrm{ac}}-\eta\right)}=-\frac{1}{2} \frac{\partial \mathrm{u}}{\partial \mathrm{x}}\left(\mathrm{r}_{\mathrm{w}}^{2}-\left(\mathrm{r}_{\mathrm{ac}}-\eta\right)^{2}\right)$.

With $\mathrm{v}=0$ at the wall and with both the remaining LHS and the RHS, of Eq. (9), divided through by $\left(r_{\mathrm{ac}}-\eta\right)$ this gives 
$-\mathrm{v}_{\left(\mathrm{r}=\mathrm{r}_{\mathrm{ac}}-\eta\right)}=-\frac{1}{2} \frac{\partial \mathrm{u}}{\partial \mathrm{x}} \frac{\left(\mathrm{r}_{\mathrm{w}}^{2}-\left(\mathrm{r}_{\mathrm{ac}}-\eta\right)^{2}\right)}{\left(\mathrm{r}_{\mathrm{ac}}-\eta\right)}$.

As an aside note here: strictly speaking $\mathrm{v}$ will only vanish at the wall if the wall is normal to v, i.e. if the wall is cylindrical. This is not the case in the conical convergence. However we are interested in the behaviour of the liquid surface at the exit, where we might, subsequent to the current work, analyse the resultant liquid break up due to these waves. Indeed Crapper [3], in his work on gravity waves to which the current article parallels, makes the point that the velocity normal to the sea bed is only in fact zero when the sea bed can be assumed to be level.

The RHS of Eq. (10) may now be used to replace the RHS of Eq. (6) to give

$\frac{\partial \eta}{\partial \mathrm{t}}+\frac{1}{2}\left[\frac{\mathrm{r}_{\mathrm{w}}^{2}-\left(\mathrm{r}_{\mathrm{ac}}-\eta\right)^{2}}{\mathrm{r}_{\mathrm{ac}}-\eta}\right] \frac{\partial \mathrm{u}}{\partial \mathrm{x}}=0$.

This will be left for the moment and attention will be turned to the pressure.

From the Bernoulli equation for inviscid flows

$\frac{\mathrm{p}}{\rho_{\mathrm{L}}}+\frac{\mathrm{w}^{2}}{2}=$ constant,

across any radial position within the annulus of liquid within the swirl nozzle (assume $\mathrm{u}$ is also constant across any cross section). Here $\mathrm{p}$ is the pressure and $\mathrm{w}$ is the tangential velocity of the spinning liquid and $\rho_{\mathrm{L}}$ is the liquid density. For a free-vortex flow

$\mathrm{w}=\frac{\mathrm{c}}{\mathrm{r}}$

where $\mathrm{c}$ is a further constant called the free-vortex constant (see ESF). At the surface of the air-core, $r=r_{a c}-\eta$, the pressure is atmospheric and is taken as zero gauge pressure, $\mathrm{p}=0$, so that Eq. (12), using Eq. (13), gives

$0+\frac{\mathrm{c}^{2}}{2\left(\mathrm{r}_{\mathrm{ac}}-\eta\right)^{2}}=$ constant

thus defining the Bernoulli equation constant for Eq. (12). This gives

$\frac{\mathrm{p}}{\rho_{\mathrm{L}}}+\frac{\mathrm{w}^{2}}{2}=\frac{\mathrm{c}^{2}}{2\left(\mathrm{r}_{\mathrm{ac}}-\eta\right)^{2}}$ or $\frac{\mathrm{p}}{\rho_{\mathrm{L}}}=\frac{\mathrm{c}^{2}}{2}\left(\frac{1}{\left(\mathrm{r}_{\mathrm{ac}}-\eta\right)^{2}}-\frac{1}{\mathrm{r}^{2}}\right)$.

The incompressible Euler equation,

$\frac{\partial \underline{\mathrm{V}}}{\partial \mathrm{t}}+\nabla\left(\frac{1}{2} \underline{\mathrm{V}}^{2}+\frac{\mathrm{p}}{\rho_{\mathrm{L}}}\right)=0$

applied in the axial direction gives $\frac{\partial u}{\partial t}+u \frac{\partial u}{\partial x}+\frac{\partial}{\partial x}\left(\frac{p}{\rho_{L}}\right)=0$

With $\mathrm{p} / \rho_{\mathrm{L}}$ as given in Eq. (15) then Eq. (17) becomes

$\frac{\partial \mathrm{u}}{\partial \mathrm{t}}+\mathrm{u} \frac{\partial \mathrm{u}}{\partial \mathrm{x}}+\frac{\mathrm{c}^{2}}{\left(\mathrm{r}_{\mathrm{ac}}-\eta\right)^{3}} \frac{\partial \eta}{\partial \mathrm{x}}=0$.

The middle term, of Eq. (18), may be omitted as it is second order in the small parameter $\varepsilon$ so that Eq. (18) reduces to

$\frac{\partial \mathrm{u}}{\partial \mathrm{t}}+\frac{\mathrm{c}^{2}}{\left(\mathrm{r}_{\mathrm{ac}}-\eta\right)^{3}} \frac{\partial \eta}{\partial \mathrm{x}}=0$.

Now, Eq. (11) may be differentiated w.r.t. $t$ and Eq. (19) differentiated w.r.t. x. With any further nonlinear terms, in the small parameter $\varepsilon$, removed (i.e. the products $\partial \eta / \partial \mathrm{t}$ $\partial \mathrm{u} / \partial \mathrm{x}$ and $\left.(\partial \eta / \partial \mathrm{t})^{2}\right)$ these reduce to

$\frac{\partial^{2} \eta}{\partial t^{2}}+\frac{1}{2}\left[\frac{\mathrm{r}_{\mathrm{w}}^{2}-\left(\mathrm{r}_{\mathrm{ac}}-\eta\right)^{2}}{\mathrm{r}_{\mathrm{ac}}-\eta}\right] \frac{\partial^{2} \mathrm{u}}{\partial \mathrm{x} \partial \mathrm{t}}=0$

and

$\frac{\partial^{2} \mathrm{u}}{\partial \mathrm{t} \partial \mathrm{x}}+\frac{\mathrm{c}^{2}}{\left(\mathrm{r}_{\mathrm{ac}}-\eta\right)^{3}} \frac{\partial^{2} \eta}{\partial \mathrm{x}^{2}}=0$.

By multiplying Eq. (21) through by the coefficient of $\partial^{2} \mathrm{u} / \partial \mathrm{x} \partial \mathrm{t}$ from Eq. (20), in square brackets, and then subtracting the result from Eq. (20), so as to remove the $\partial^{2} \mathrm{u} / \partial \mathrm{x} \partial \mathrm{t}$ terms, then one obtains

$\frac{\partial^{2} \eta}{\partial \mathrm{t}^{2}}-\left[\frac{\mathrm{c}^{2}\left\{\mathrm{r}_{\mathrm{w}}^{2}-\left(\mathrm{r}_{\mathrm{ac}}-\eta\right)^{2}\right\}}{2\left(\mathrm{r}_{\mathrm{ac}}-\eta\right)^{4}}\right] \frac{\partial^{2} \eta}{\partial \mathrm{x}^{2}}=0$.

As a coefficient, the wave amplitude $\eta$ is small in comparison to both the air-core radius $r_{a c}$ and the nozzle wall radius $r_{w}$ then the coefficient of $\partial^{2} \eta / \partial x^{2}$ may be approximated to give

$\frac{\partial^{2} \eta}{\partial \mathrm{t}^{2}}-\left[\frac{c^{2}\left\{\mathrm{r}_{\mathrm{w}}^{2}-\mathrm{r}_{\mathrm{ac}}^{2}\right\}}{2 \mathrm{r}_{\mathrm{ac}}^{4}}\right] \frac{\partial^{2} \eta}{\partial \mathrm{x}^{2}}=0$.

or

$\frac{\partial^{2} \eta}{\partial \mathrm{t}^{2}}-\chi^{2} \frac{\partial^{2} \eta}{\partial \mathrm{x}^{2}}=0$

This is a wave equation with the wave phase velocity given by

$\chi= \pm\left(\frac{\mathrm{c}^{2}\left(\mathrm{r}_{\mathrm{w}}^{2}-\mathrm{r}_{\mathrm{ac}}^{2}\right)}{2 \mathrm{r}_{\mathrm{ac}}^{4}}\right)^{1 / 2}$

This will be referred to at the end of the next section. 


\section{The principle of maximum flow}

The flow domain for this analysis is a convergent and effectively divergent swirl nozzle shown in Fig. 1, where the swirling flow is moving from left to right. The differential between the stagnation pressure, in some supply reservoir, and that at the air-core, at which the pressure is zero gauge, is $\Delta \mathrm{p}$. The usual supposition regarding the radial velocity $\mathrm{v}$ is that it is negligible in comparison to the swirl and axial velocities ( $\mathrm{w}$ and $\mathrm{u}$, respectively). The radial velocity $\mathrm{v}$, of the bulk flow, will certainly be small adjacent to the air-core.

The analysis is in two parts. In the first part, an expression for the axial velocity $u_{c}$ through the 'throat' or constriction (in practice, the outlet) is determined by putting the axial change in the volumetric flow equal to zero, $\partial \mathrm{Q} / \partial \mathrm{x}=0$, by continuity. In the second part an expression is derived for $\partial \mathrm{Q} / \partial \mathrm{r}_{\mathrm{ac}}$ at the throat; where $r_{a c}$ is the radius of the air-core at any axial position, $\mathrm{x}$, within the nozzle. It is then shown that by putting the expression for $u_{c}$ (the 'critical velocity'), obtained in the first part, into this expression for $\partial \mathrm{Q} / \partial \mathrm{r}_{\mathrm{ac}}$, obtained in the second part, that indeed $\partial \mathrm{Q} / \partial \mathrm{r}_{\mathrm{ac}}=0$ at the throat. Thus, the aircore in the throat will adjust itself so as to permit maximum flow. This is then a direct analogy of the flow over a weir (see ESF) where the water depth over the crest of the weir adjusts itself so that the flow is a maximum.

\subsection{First part}

The Bernoulli equation, for this flow, is

$$
\frac{\mathrm{u}^{2}}{2}+\frac{\mathrm{w}^{2}}{2}+\frac{\mathrm{p}}{\rho_{\mathrm{L}}}=\frac{\Delta \mathrm{p}}{\rho_{\mathrm{L}}} \text {. }
$$

Here $\rho_{\mathrm{L}}$ is the liquid density. By continuity, the mean axial velocity across any cross-section of the nozzle will be given by

$$
\begin{gathered}
\mathrm{u}=\frac{\mathrm{Q}}{\pi\left(\mathrm{r}_{\mathrm{w}}^{2}-\mathrm{r}_{\mathrm{ac}}^{2}\right)} . \\
\mathrm{wr}=\mathrm{w}_{\mathrm{ac}} \mathrm{r}_{\mathrm{ac}}=\mathrm{c} .
\end{gathered}
$$

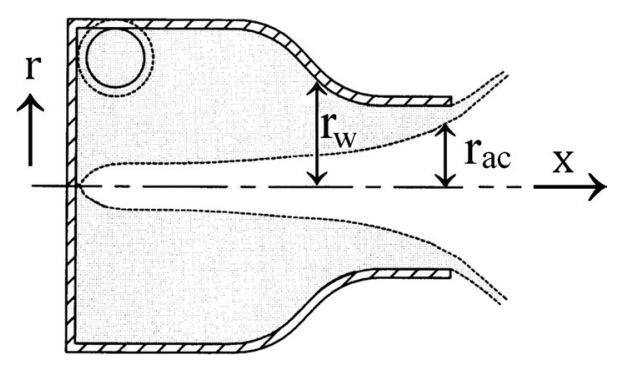

Fig. 1 A cross-section of the flow domain a typical, single tangential inlet, swirl atomizer showing the air-core and the variables: the wall radius $r_{w}$, and the air-core radius $r_{a c}$
For irrotational flow, the swirl velocity is given by a free vortex (see ESF) so that, at the air-core within the nozzle:

At the air-core, $\mathrm{p}=0$ so that on using Eq. (28) for $\mathrm{w}_{\mathrm{ac}}$ then Eq. (26) may be rearranged to form an expression for $\mathrm{u}$ :

$\mathrm{u}=\left(\frac{2 \Delta \mathrm{p}}{\rho_{\mathrm{L}}}-\frac{\mathrm{c}^{2}}{\mathrm{r}_{\mathrm{ac}}^{2}}\right)^{1 / 2}$.

Next, Eq. (27) is arranged to make $Q$ the subject and is then differentiated w.r.t. $\mathrm{x}$, the axial coordinate, to form

$$
\frac{\partial \mathrm{Q}}{\partial \mathrm{x}}=2 \pi \mathrm{u}\left(\mathrm{r}_{\mathrm{w}} \frac{\mathrm{dr}_{\mathrm{w}}}{\mathrm{dx}}-\mathrm{r}_{\mathrm{ac}} \frac{\mathrm{dr}_{\mathrm{ac}}}{\mathrm{dx}}\right)+\pi\left(\mathrm{r}_{\mathrm{w}}^{2}-\mathrm{r}_{\mathrm{ac}}^{2}\right) \frac{\partial \mathrm{u}}{\partial \mathrm{x}}=0 .
$$

This is zero, as the volumetric flow rate $\mathrm{Q}$ is continuous throughout the nozzle. Equation (29) may also be differentiated w.r.t. to $\mathrm{x}$ to form

$$
\frac{\partial u}{\partial x}=\left(\frac{2 \Delta p}{\rho_{L}}-\frac{c^{2}}{r_{a c}^{2}}\right)^{-1 / 2} \frac{c^{2}}{r_{a c}^{3}} \frac{\partial r_{a c}}{\partial x} \text { or } u \frac{\partial u}{\partial x}=\frac{c^{2}}{r_{a c}^{3}} \frac{\partial r_{a c}}{\partial x} .
$$

Equation (30) may be rearranged to make $\mathrm{dr}_{\mathrm{ac}} / \mathrm{dx}$ the subject and the resulting expression may be substituted for $\mathrm{dr}_{\mathrm{ac}} / \mathrm{dx}$ into Eq. (31) to give

$\mathrm{u} \frac{\partial \mathrm{u}}{\partial \mathrm{x}}=\frac{\mathrm{c}^{2}}{\mathrm{r}_{\mathrm{ac}}^{3}}\left(\frac{\left(\mathrm{r}_{\mathrm{w}}^{2}-\mathrm{r}_{\mathrm{ac}}^{2}\right)}{2 \mathrm{r}_{\mathrm{ac}}} \frac{\partial \mathrm{u}}{\partial \mathrm{x}}+\frac{\mathrm{r}_{\mathrm{w}}}{\mathrm{r}_{\mathrm{ac}}} \frac{\mathrm{dr}_{\mathrm{w}}}{\mathrm{dx}}\right)$.

The grouping of the coefficients of $\mathrm{du} / \mathrm{dx}$ in the above yields

$\frac{\partial u}{\partial x}\left(\frac{u^{2}-u_{c}^{2}}{u}\right)-\frac{c^{2} r_{w}}{r_{a c}^{4}} \frac{d r_{w}}{d x}=0$,

where $u_{c}$ is defined as

$\mathrm{u}_{\mathrm{c}}^{2}=\frac{\mathrm{c}^{2}\left(\mathrm{r}_{\mathrm{w}}^{2}-\mathrm{r}_{\mathrm{ac}}^{2}\right)}{2 \mathrm{r}_{\mathrm{ac}}^{4}}$.

At the throat (outlet) $\partial \mathrm{r}_{\mathrm{w}} / \partial \mathrm{x}=0$, but $\partial \mathrm{u} / \partial \mathrm{x} \neq 0$ because the annulus of liquid continues to become thinner with $\mathrm{x}$ as it passes through the outlet (as shown in Fig. 1) which in turn causes u to continue to increase. Therefore, in order for Eq. (33) to be equal to zero then it only remains that the axial velocity $\mathrm{u}$ must be equal to $u_{c}$, as given in Eq. (34), where $r_{w}$ and $r_{a c}$ take their respective values at the throat: $\mathrm{r}_{\mathrm{w}}\left(\mathrm{x}_{\mathrm{c}}\right)$ and $\mathrm{r}_{\mathrm{ac}}\left(\mathrm{x}_{\mathrm{c}}\right)$.

\subsection{Second part}

The second part of this analysis is designed to demonstrate the principle of maximum flow for a swirl nozzle by 
putting $\partial \mathrm{Q} / \partial \mathrm{r}_{\mathrm{ac}}=0$. This parallels the weir flow analysis (see ESF) where the derivative of the discharge (volumetric flow rate) w.r.t the height of the weir, $\mathrm{h}(\mathrm{x}), \partial \mathrm{Q} / \partial \mathrm{h}$ is set equal to zero. Equation (27) is again rearranged to make $\mathrm{Q}$ the subject and the resulting expression is differentiated, this time w.r.t. $r_{\mathrm{ac}}$, giving

$\frac{\partial \mathrm{Q}}{\partial \mathrm{r}_{\mathrm{ac}}}=-2 \mathrm{u} \pi \mathrm{r}_{\mathrm{ac}}+\pi\left(\mathrm{r}_{\mathrm{w}}^{2}-\mathrm{r}_{\mathrm{ac}}^{2}\right) \frac{\partial \mathrm{u}}{\partial \mathrm{r}_{\mathrm{ac}}}$.

Equation (29) is also differentiated w.r.t. $r_{\text {ac }}$ which results in

$\frac{\partial \mathrm{u}}{\partial \mathrm{r}_{\mathrm{ac}}}=\left(\frac{2 \Delta \mathrm{p}}{\rho_{\mathrm{L}}}-\frac{\mathrm{c}^{2}}{\mathrm{r}_{\mathrm{ac}}^{2}}\right)^{-1 / 2} \frac{\mathrm{c}^{2}}{\mathrm{r}_{\mathrm{ac}}^{3}} \quad$ or $\quad \frac{\partial \mathrm{u}}{\partial \mathrm{r}_{\mathrm{ac}}}=\frac{1}{u} \frac{\mathrm{c}^{2}}{\mathrm{r}_{\mathrm{ac}}^{3}}$.

By substituting this expression for $\partial \mathrm{u} / \partial \mathrm{r}_{\mathrm{ac}}$ into Eq. (35) one obtains, on simplification,

$\frac{\partial \mathrm{Q}}{\partial \mathrm{r}_{\mathrm{ac}}}=\frac{2 \pi \mathrm{r}_{\mathrm{ac}}}{\mathrm{u}}\left(\frac{\mathrm{c}^{2}\left(\mathrm{r}_{\mathrm{w}}^{2}-\mathrm{r}_{\mathrm{ac}}^{2}\right)}{2 \mathrm{r}_{\mathrm{ac}}^{4}}-\mathrm{u}^{2}\right)$.

At the throat $u$ must be equal to $u_{c}$, as given by Eq. (34), which was derived by the sound reasoning that the volumetric flow, by continuity, will not vary with axial distance, i.e. $\partial \mathrm{Q} / \partial \mathrm{x}=0$, and then Eq. (37) gives that, at the throat, $\partial \mathrm{Q} / \partial \mathrm{r}_{\mathrm{ac}}=0$. Thus the air-core radius at the throat, $\mathrm{r}_{\mathrm{ac}}\left(\mathrm{x}_{\mathrm{c}}\right)$, must adjust itself so that $\mathrm{Q}$ is a maximum. In practice, as $\mathrm{Q}$ is a constant, this means that the air-core radius and the axial velocity within the outlet will adjust themselves optimally. This is the principle of maximum flow. Equation (34) is both an expression for the critical velocity within the outlet of the swirl atomizer and the wave phase velocity of a shallow 'rotary force wave' occurring on the surface of the air-core. Compare with Eq. (25) from Sect. 2

$\mathrm{u}=\mathrm{u}_{\mathrm{c}}=\chi=\left(\frac{\mathrm{c}^{2}\left(\mathrm{r}_{\mathrm{w}}^{2}-\mathrm{r}_{\mathrm{ac}}^{2}\right)}{2 \mathrm{r}_{\mathrm{ac}}^{4}}\right)^{1 / 2}$.

\section{The critical velocity as the square root of the film thickness and rotational acceleration}

For weir flow, and shallow water gravity waves the critical velocity over the weir and the wave phase velocity are both given by

$\mathrm{u}_{\mathrm{c}}=\chi=\sqrt{\mathrm{gh}}$,

i.e. the square root of the product of the gravitational acceleration and the water depth, h, over the weir (see ESF). Similarly, Eq. (38) can be shown to be the square root of the product of the centrifugal acceleration and the annular liquid thickness in the atomizer outlet: $\frac{\mathrm{w}_{\mathrm{ac}}^{2}}{\mathrm{r}_{\mathrm{ac}}}$ and $\mathrm{r}_{\mathrm{w}}-\mathrm{r}_{\mathrm{ac}}$.

From Eq. (28), using $\mathrm{c}=\mathrm{w}_{\mathrm{ac}} \mathrm{r}_{\mathrm{ac}}$, and expanding $\mathrm{r}_{\mathrm{w}}^{2}-\mathrm{r}_{\mathrm{ac}}^{2}$ Eq. (38) may be written as

$\mathrm{u}_{\mathrm{c}}=\chi=\left(\frac{\mathrm{w}_{\mathrm{ac}}^{2} \mathrm{r}_{\mathrm{ac}}^{2}\left(\mathrm{r}_{\mathrm{ac}}+\mathrm{r}_{\mathrm{ac}}\right)\left(\mathrm{r}_{\mathrm{w}}-\mathrm{r}_{\mathrm{ac}}\right)}{2 \mathrm{r}_{\mathrm{ac}}^{4}}\right)^{1 / 2}$.

At the outlet, the annulus of liquid is thin and $r_{w}$ and $r_{a c}$ are of similar magnitude so that Eq. (41) may be approximated by

$\mathrm{u}_{\mathrm{c}}=\mathrm{x} \approx\left(\frac{\mathrm{w}_{\mathrm{ac}}^{2} \mathrm{r}_{\mathrm{ac}}^{2}\left(\mathrm{r}_{\mathrm{w}}+\mathrm{r}_{\mathrm{ac}}\right)\left(\mathrm{r}_{\mathrm{w}}-\mathrm{r}_{\mathrm{ac}}\right)}{2 \mathrm{r}_{\mathrm{ac}}^{4}}\right)^{1 / 2}=\left(\frac{\mathrm{w}_{\mathrm{ac}}^{2}\left(\mathrm{r}_{\mathrm{w}}-\mathrm{r}_{\mathrm{ac}}\right)}{\mathrm{r}_{\mathrm{ac}}}\right)^{1 / 2}$.

This is in the form of the product of the centripetal acceleration and the liquid thickness in the outlet, as suggested in Eq. (40).

\section{Experiments on a large scale model swirl atomizer using high speed video, LDA and deflected laser beam}

\subsection{Apparatus}

\subsubsection{Plexiglass model swirl atomizer}

The atomizer was constructed from Perspex in modular form so that the number of inlet ports, length of swirl chamber and outlet geometry could be changed independently, Fig. 2. The length of the swirl chamber and the outlet geometry were kept constant and two and eight inlet ports were used. The working fluid was water supplied to the atomizer from a holding tank by means of a centrifugal pump via a flowmeter. The working fluid was fed into a plenum chamber immediately above the atomizer body so that any swirling motion of the water and any entrained air could be removed.

\subsubsection{Camera system}

The camera used was a Kodak Ectapro HS Motion Analyser 4540 video camera fitted with a Cannon J6 $\times 11$, $11-70 \mathrm{~mm}$ zoom lens. Full frame $(750 \times 540$ pixel $)$ images were obtained at $4500 \mathrm{fps}$ over $0.683 \mathrm{~s}$. Individual frames were then imported into suitable software for analysis. Lighting was by means of two $500 \mathrm{~W}$ Halogen spotlights fitted with diffuser screens situated behind and slightly to either side of the atomizer. 
Fig. 2 A cross-section of the large scale Perspex model atomizer. This example shows two rectangular inlets and a conical convergence

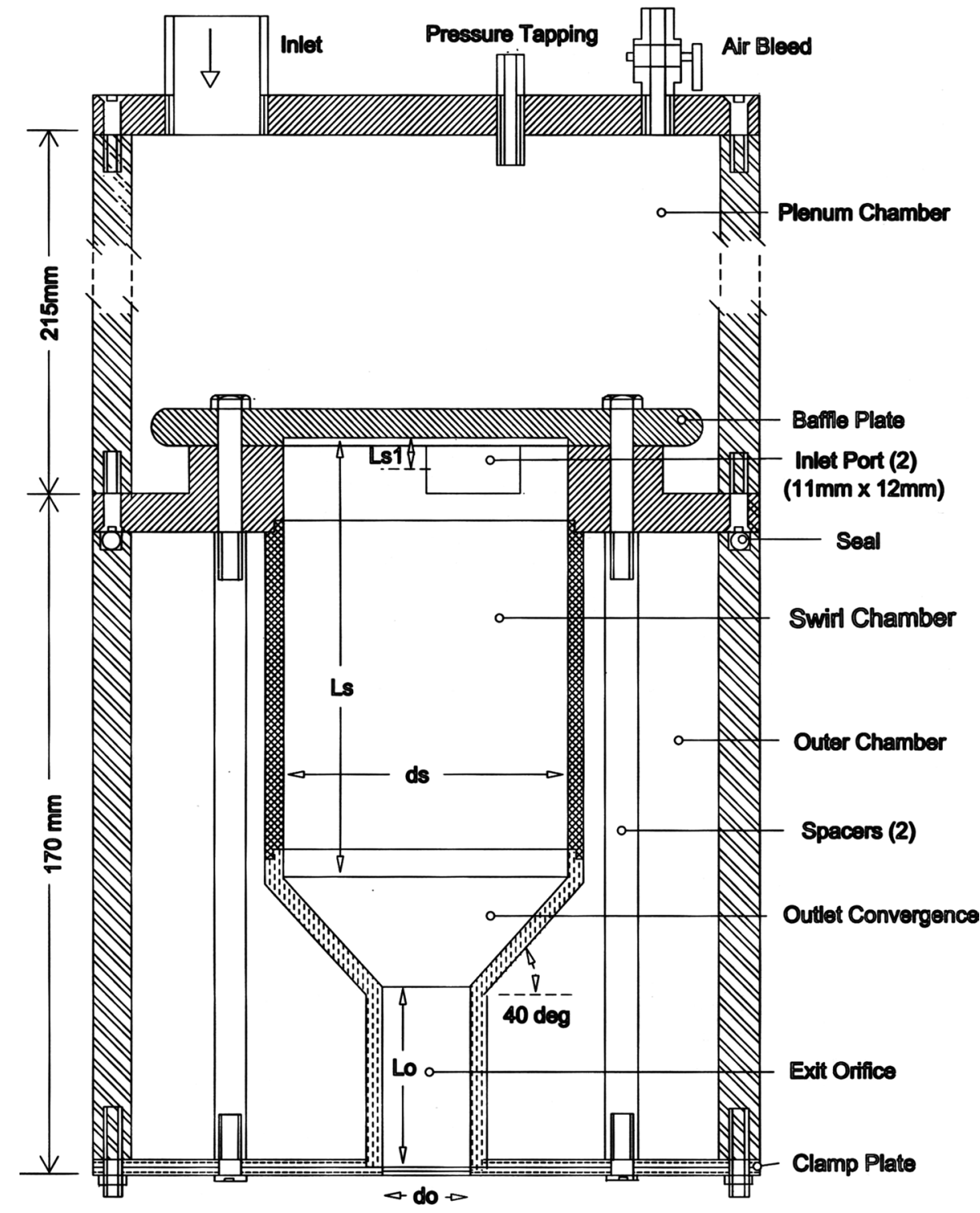

\subsubsection{Laser system}

Two different laser techniques were used, the first technique used was LDA by means of a Dantec 'Classic' Phase Doppler Anemometer (PDA) system, this system can measure both droplet size and velocity but for the current work the sizing facility was turned off and the system was used only in velocity mode. A 2D LDA (or PDA) system works by transmitting a pair of converging laser beams so that they cross at a certain focal length and form a Measurement Control Volume (MCV). Within the MCV optical interference fringes are generated where the distance between fringes can be calculated from $\lambda / 2 \sin \theta$ where $\lambda$ is the wavelength of the light $(512 \mathrm{~nm}$, green, in the present case), and $\theta$ is the half-angle. Particles in the flow crossing through the MCV (and through each interference fringe) produces pulses of light which are timed so now we have both distance and time $=$ velocity. The direction of flow can be determined by superimposing a Doppler Shift Frequency $(40 \mathrm{MHz})$ on one of the beams. For the ' $u$ ' component of velocity the laser beams were orientated on the vertical plane and corrected for any change in the location of the MCV due to refraction caused by the change in density between the surrounding air, atomiser wall and working fluid while for the ' $w$ ' component the beams were rotated by $90^{\circ}$ to the horizontal plane and also corrected for refraction caused not only by density changes but also for effects of the curvature of the atomiser wall, traversing was undertaken in the radial $(z)$ direction. To ensure that near-wall measurements could be obtained reliably without excessive light scattering the receiving optics were setup in $30^{\circ}$ off-axis backscatter mode and a spatial filter was used to minimise the effective MCV size. Aluminium powder, as used in metallic paints, was used as seeding because the particles are the correct size, avg $5 \mu \mathrm{m}$ dia, and reflect light very efficiently. 


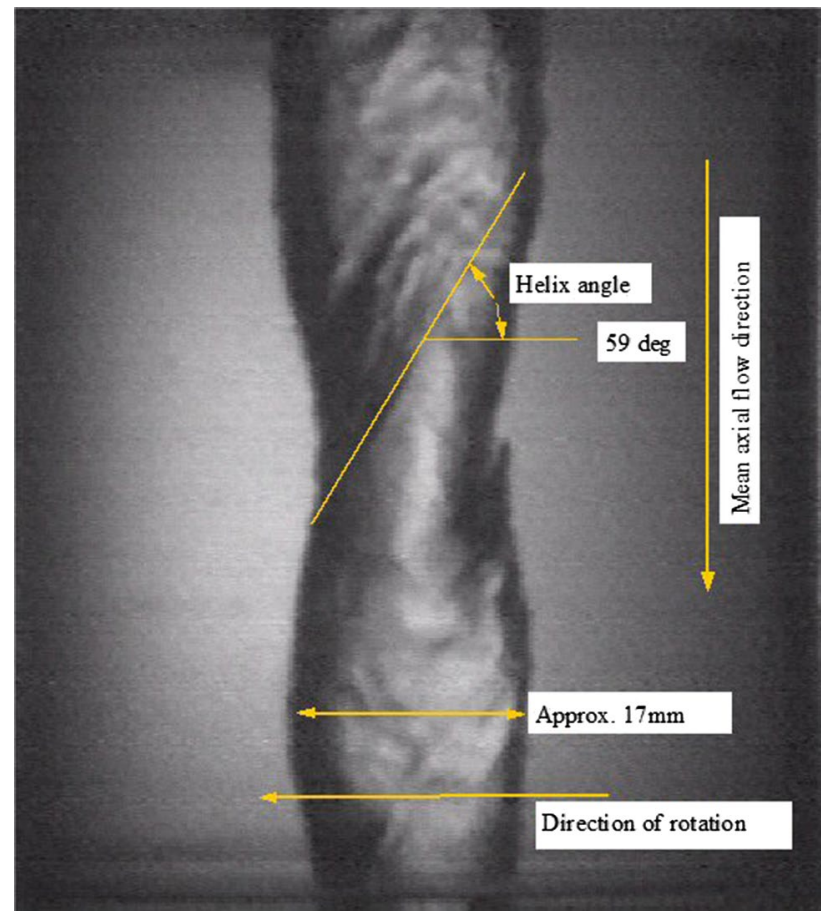

Fig. 3 Air core in swirl chamber, 2 inlets

For the second technique, deflected laser beam, the beam from a Helium Neon laser was positioned so as to just strike the edge of the air core and the presence of waves on the air core would then deflect the laser beam. The single laser beam is only deflected onto the detector when an expansion of the air core is present, for a contraction of the air core the laser beam does not impinge on the detector. The deflected beam swept across a lens with a focal length of $150 \mathrm{~mm}$ that focused the laser light on to a pinhole in front of a photodetector. The result of this was to produce a series of electrical pulses at the output of the photodetector, each pulse representing the presence of a 'wave'. The data were captured by means of a high speed ADC connected to a computer, and was analysed by means of a FFT routine to give a frequency spectra. Prior to utilising this technique the system was checked by 'bouncing' a laser beam off a small loudspeaker cone that was driven by a signal generator with a pure sine wave over the range of frequencies that were expected in the experimental work, received signals were better than $1 \%$ accurate compared to the input signal.

\subsection{Experimental results}

\subsubsection{Helical striation waves}

Figure 3 shows one video frame of the air core within the swirl chamber at a flow rate of $0.212 \mathrm{l} / \mathrm{s}$. This low flow rate was insufficient to allow a full spray cone to

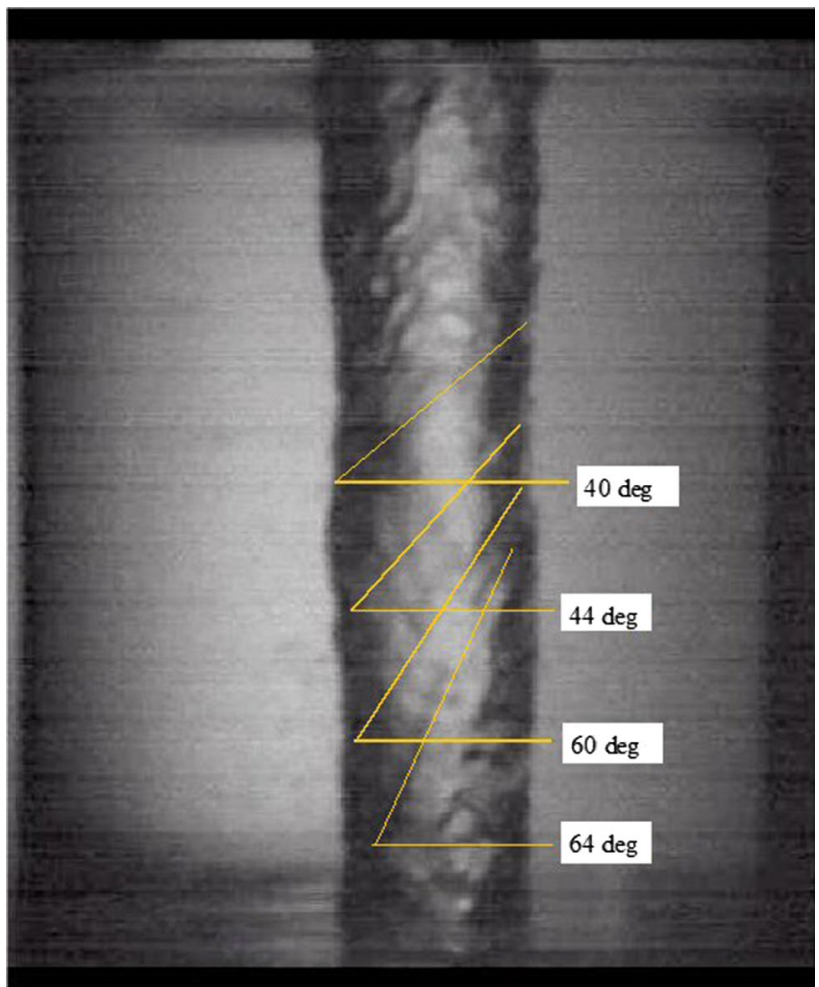

Fig. 4 Air core in swirl chamber, 8 inlets

develop, however it illustrates the different physical aspects of events on or near the air core. This particular air core was generated by means of the two-port inlet and it was clearly seen that the two discreet inlet flows form a double helix. The double helix is rotating in the same sense as the swirl in the chamber, giving the localised appearance of surface waves moving upstream, away from the outlet. For the 2 inlet atomizer, at this low flow rate, the helix angle varies insignificantly throughout the swirl chamber. Figure 4 shows the same view as Fig. 3, with the same flow rate, but this time there were 8 inlet ports. It can be seen that the 'double helix' form of Fig. 3 has changed and although it is not clear exactly how many 'streaks' are visible there seems likely to be, on average, eight. It is clear, for the 8 inlet case, that the helix angle changes as the flow moves downstream. This shows that the two inlets feed the flow into the air core while the eight inlets "spreads" the flow more uniformly at the inlet. Below a flow rate of $0.4 \mathrm{l} / \mathrm{s}$, increasing the flow gives a shallower helix angle and a larger spray cone angle. However, above $0.4 \mathrm{l} / \mathrm{s}$ increasing the flow, still decreases the helix angle at any given position within the swirl chamber, which appears to be the same for both 2 and 8 port cases, while leaving the spray cone angle almost unaffected. Typically, for a flow rate of $0.428 \mathrm{l} / \mathrm{s}$ the air core helix angle within the swirl chamber is $27^{\circ}$ 
Fig. 5 Two video frames of air core in swirl chamber, $0.038 \mathrm{~s}$ apart, $0.4281 / \mathrm{s}$

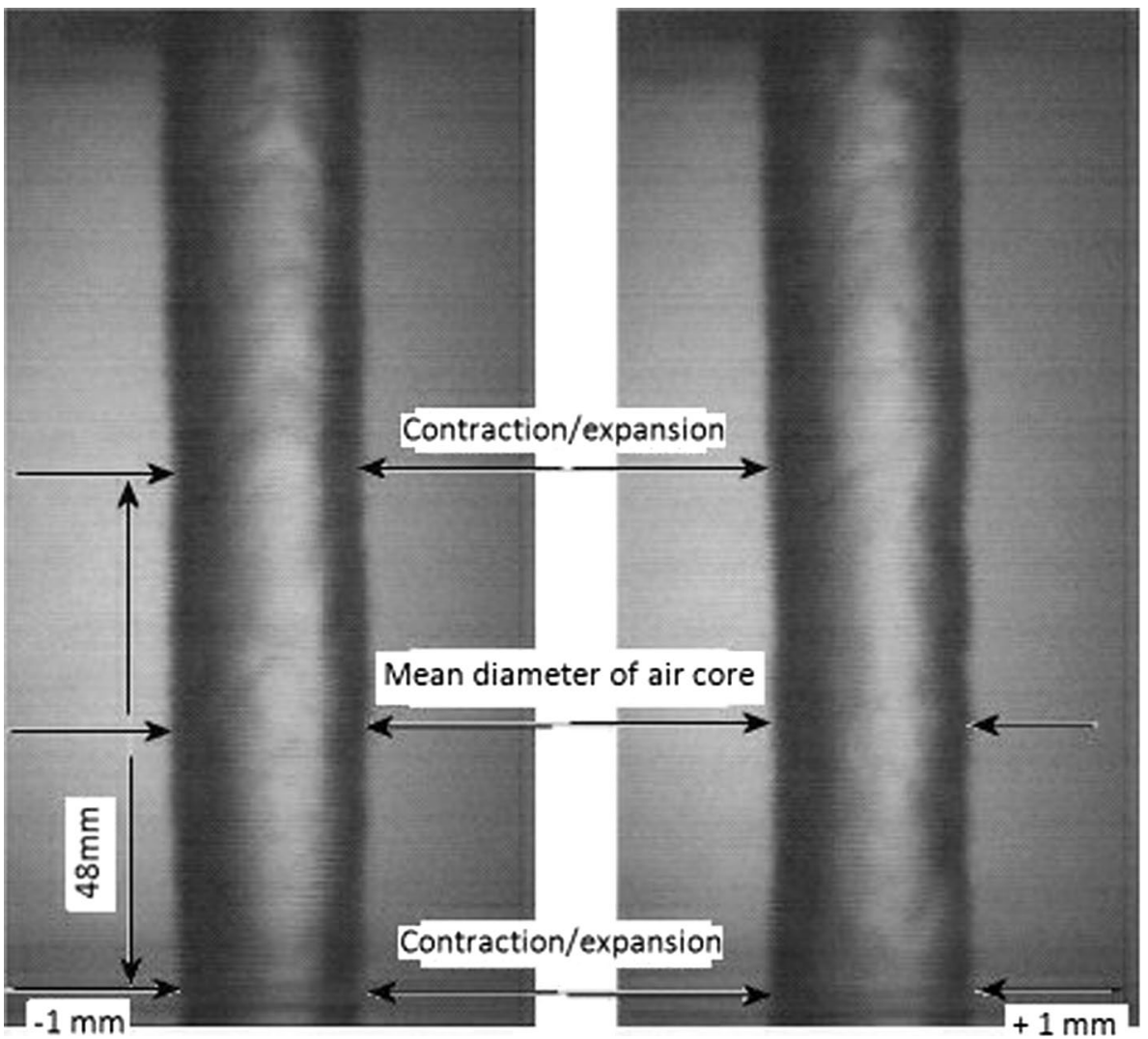

at $50 \mathrm{~mm}$ before the outlet, increasing to $45^{\circ}$ at $22 \mathrm{~mm}$ before the outlet.

\subsubsection{Stationary pulsating waves}

For both 2 and 8 inlets, and quite separate from the helical waves, observations have been made of a phenomenon whereby localised regions of the air core are seen to expand and contract with a regular period. These expansion/contraction regions appear in two locations on the air core/ liquid interface and are spaced approximately $48 \mathrm{~mm}$ apart as can be seen in Fig. 5 and they will be referred to as the "stationary waves". The mean streamline plot is shown in Fig. 6, for the case with the conical convergence. It is recalled that no information is shown in these figures on the swirl component, w. It is only in the near-air core zone that the axial, $u$ component becomes comparable with the swirl component. The streamlines in the figures are actually surface streamtraces i.e. cross-sections of 3-D stream tubes plotted on a 2-D surface, they were calculated from the $\mathrm{u}$ and $v$ values, obtained by LDA measurements, using TECPLOT $^{\circledR}$. TECPLOT ${ }^{\circledR}$ uses a predictor-corrector integration algorithm to calculate streamtraces which are the paths traced by massless particles in the steady state vector field.

LDA data of the axial, $u$, velocity component plotted as iso-contours are shown in Fig. 7. This is a closer view of the nozzle in Fig. 6 and shares the same axial and radial coordinates. The semi-circular contour lines on the left hand vertical axis between $16 \mathrm{~mm}$ and $22 \mathrm{~mm}$ from the outlet indicate the presence of the stationary wave. It was originally postulated, before the present analysis, that this may indicate the existence of an asymmetric vortice that rotates, or precesses, about the axis. Certainly this corroborates the presence of vortices in general, as advocated by Yule and Chinn [17]. The shape of the stream tubes in Fig. 6 also show the stationary wave motion. Examination of a number of video frames indicates that the interval between expansion and contraction is approximately $0.038 \mathrm{~s}$ giving a frequency of $13 \mathrm{~Hz}$ at $0.428 \mathrm{l} / \mathrm{s}$ flow rate, the frequency increasing as the flow rate increases. As a check on this an FFT was performed on one set of the LDA tangential, w, component data to extract the frequency spectrum. This is shown in Fig. 8 and it can be seen that there is a main peak at $12 \mathrm{~Hz}$, which accords with the frequency obtained from the video, together with contributions due to the helical waves. The time period obtained from the high speed video is the time between contraction and expansion which is half the time period between each expansion therefore the frequency will be double $(0.038=1 / 26)$.

Figure 9, gives consecutive frames showing of the spray cone at $0.428 \mathrm{l} / \mathrm{s}$. On the left hand edge can be seen a number of relatively long wavelength waves (W1-W4). The 


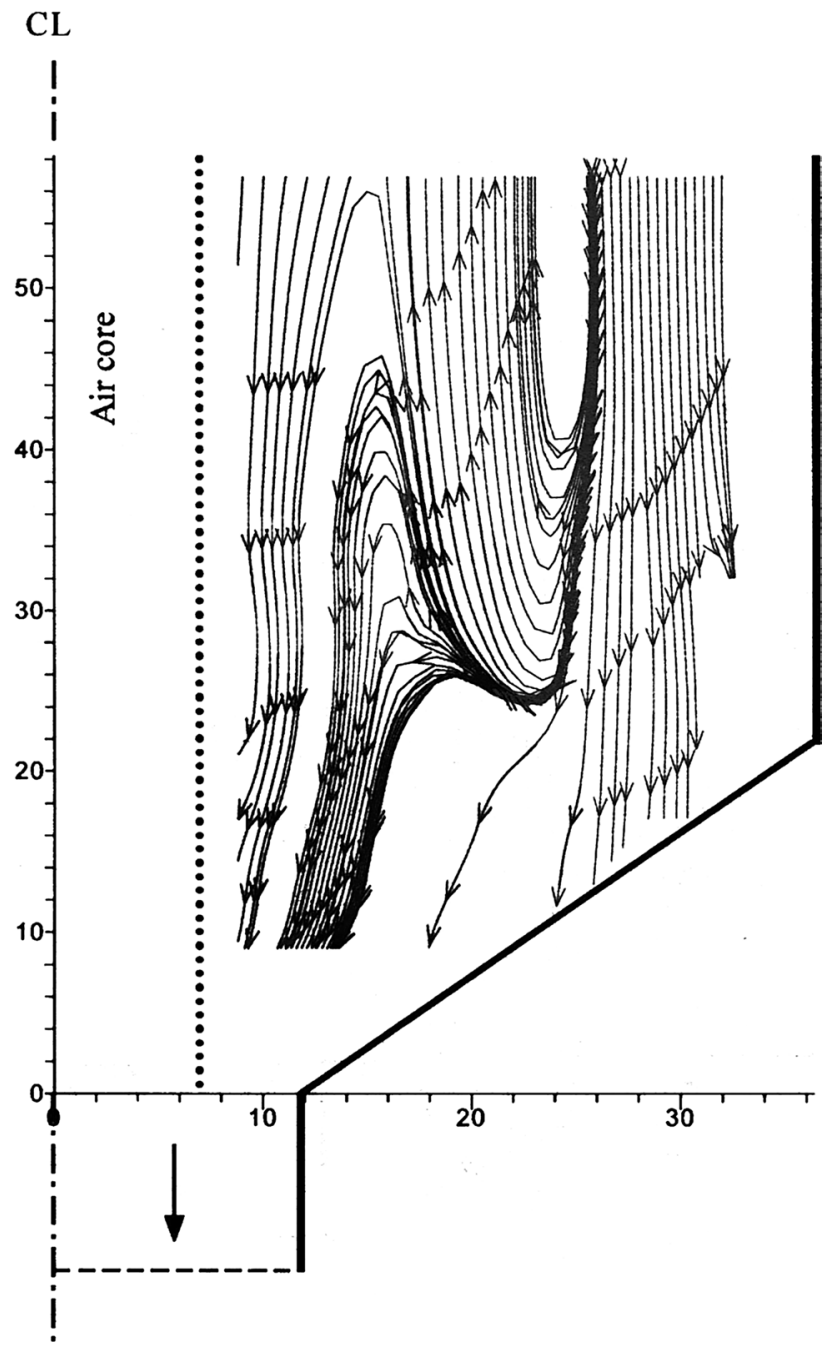

Fig. 6 Streamlines of $u$ and v velocity, 0.428 1/s

distance between $\mathrm{W} 1$ and $\mathrm{W} 2$ is $10.5 \mathrm{~mm}$, between $\mathrm{W} 2$ and W3 is $9.4 \mathrm{~mm}$ and between W3 and W4 is $8.73 \mathrm{~mm}$ which indicates that the spray cone is expanding. The time interval between frames is $2.6 \mathrm{~ms}$ and the distance travelled by the waves in this time is $9.508 \mathrm{~mm}$ giving an average velocity of $3.4 \pm 0.3 \mathrm{~m} / \mathrm{s}$ for the convection of the waves, an average passing interval of $0.035 \pm 0.006 \mathrm{~s}$ and an average frequency of $14.3 \pm 2.3 \mathrm{~Hz}$. It is believed that these waves are the results of the varying liquid film thickness in the exit orifice, which itself is a result of the local contraction/ expansion of regions on the air core.

\subsubsection{Small amplitude capillary waves}

When using the laser beam deflection method, one initial problem was that the signal was contaminated by 'noise' caused by low amplitude, random ripples on the surface

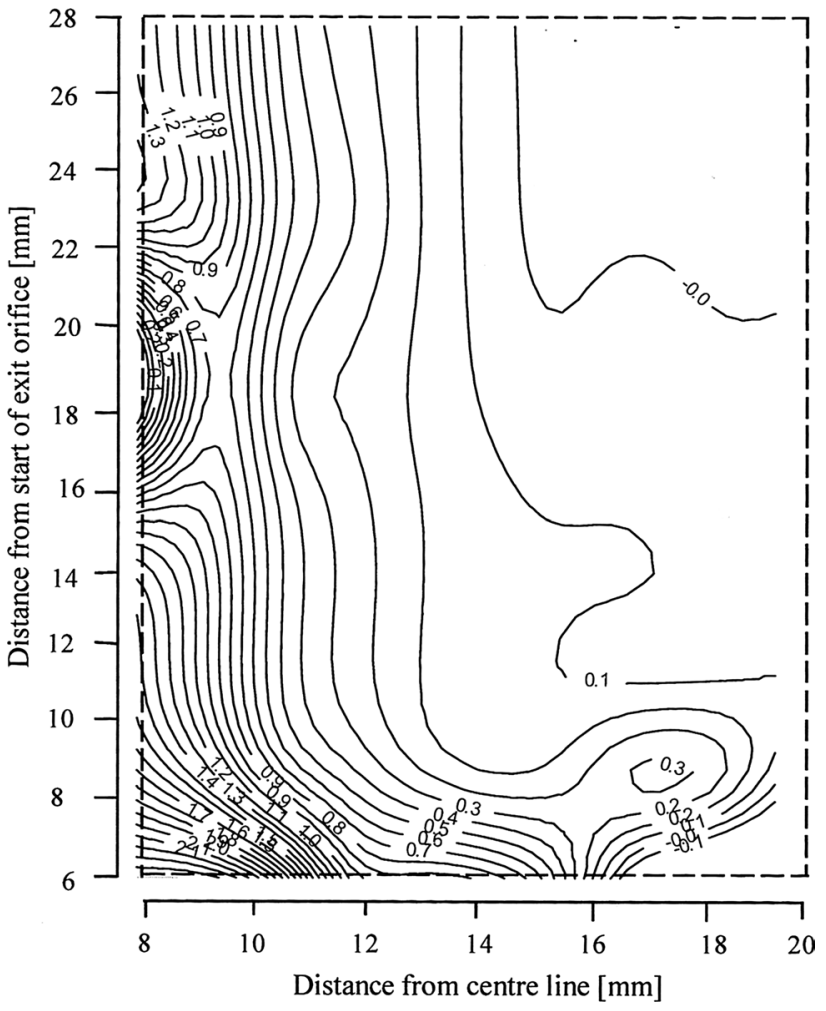

Fig. 7 Streamlines of $u$ and v velocity, 0.428 1/s

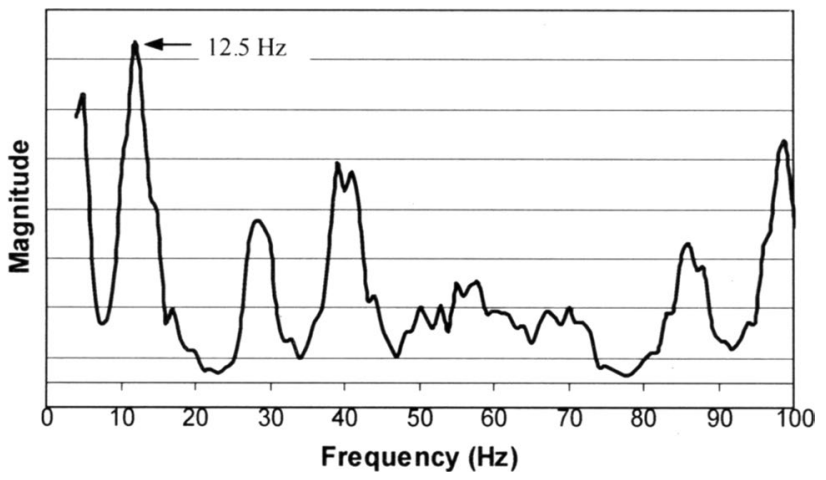

Fig. 8 Frequency spectra of W fluctuations at the air core 0.428 1/s

of the air core. The origin of these is uncertain but it was originally postulated that they may be associated with a turbulent air core/liquid interface. To remove this contamination the signal was filtered by means of a MathCad routine then a FFT was performed to extract the frequency spectra. As shown in Figs. 10 and 11, for the flow rate of 0.290 1/s it can be seen that there is a main peak at $9 \mathrm{~Hz}$ with a second peak $57 \mathrm{~Hz}$. For the $0.428 \mathrm{l} / \mathrm{s}$ flow rate there is a main peak at $12 \mathrm{~Hz}$ and a smaller second peak at $68 \mathrm{~Hz}$. Looking at these two spectra (the trend continues with lower or 

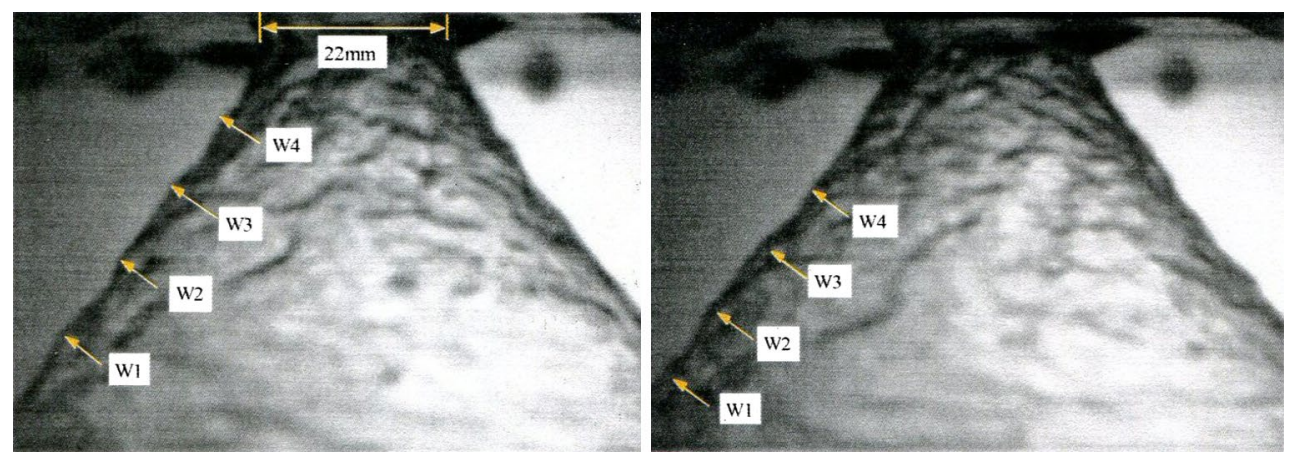

Fig. 9 Waves on the spray cone, 0.428 1/s, 2 inlets, time interval $2.6 \mathrm{~ms}$

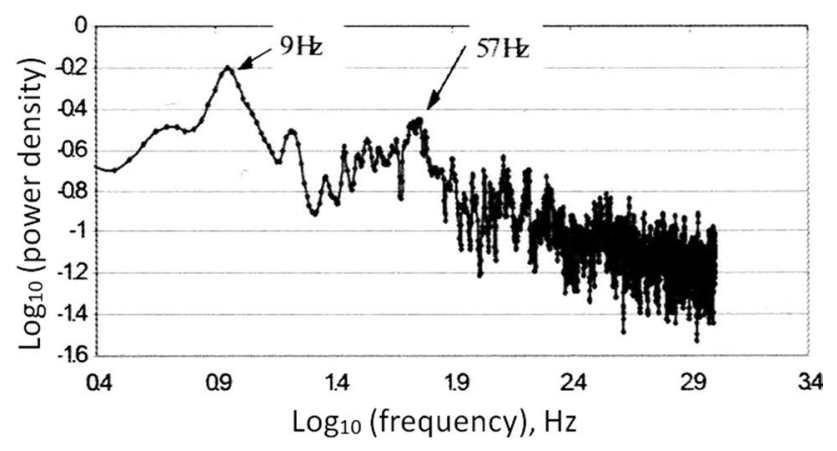

\subsection{Other observations}

\subsubsection{Flow bifurcation}

Figure 6 indicates that the highest magnitudes of the velocity components are found near the air core, with the second highest values in the near wall region. The streamlines indicate that the flow takes an ' $S$ ' pattern with a region near to the swirl chamber wall flowing downstream from the inlet to the outlet. A portion of the flow then reverses and flows back upstream but then reverses again and once again flows downstream but this time in a region adjacent to the air-core. This agrees with the predictions of Taylor [20] and with the findings of Yule and Chinn [17] and also with the measurements of Horvay and Leuckel [21], that the flow bifurcates at the inlet with two axial flow regions (a near wall region and a flow across the rear of the swirl chamber then parallel to the air-core downstream to the exit). The present work can be seen to be consistent with this. More recent numerical modelling by Nouri-Borujerdi and Kebriacee [19] confirm this flow bifurcation within the swirl chamber.

To establish if the idea of a bifurcating flow is valid with the limited data available in the present work a mass volumetric integration was performed across one specific plane of the swirl chamber, $\mathrm{x}=32 \mathrm{~mm}$ which was chosen because this plane clearly displayed distinctly different flow direction regions for all three outlet configurations.

$\mathrm{Q}_{\mathrm{x}=32}=2 \pi \sum\left[\left(\frac{\mathrm{u}_{\mathrm{j}}+\mathrm{u}_{\mathrm{j}+1}}{2}\right)\left(\mathrm{r}_{\mathrm{j}+1}-\mathrm{r}_{\mathrm{j}}\right)\left(\frac{\mathrm{r}_{\mathrm{j}}+\mathrm{r}_{\mathrm{j}+1}}{2}\right)\right]$.

higher flowrates) it appears that, taking any one significant spectral peak, the frequency increases with flow rate. The frequency peak at $12 \mathrm{~Hz}$ agrees with that obtained by high speed video, Fig. 5. The higher peak appears to be the result of the rotating double helix wave. These results concur with those of Donjat et al. [18] who also found two fundamental spectral frequencies and also mention the presence of capillary waves, in addition to the helical and air core precession, based on numerical results and laser spectrum analysis.

where $\mathrm{j}$ represents the $\mathrm{jth}$ data point measured radially from the centreline. The results gave a total volumetric flow rate of $0.406 \mathrm{l} / \mathrm{s}$ which is $95 \%$ of the total volumetric flow at the inlet ports as measured by the calibrated flowmeter. This is remarkably good agreement and supports the good accuracy of the measurements and data processing techniques. 
The second step was to calculate the volumetric flow in each distinct flow region. This operation was performed by splitting the plane into three regions, the near wall region where the flow was downstream (inlet to exit), the centre region where the flow was predominately upstream (exit to inlet) and the near-air core region where the flow was downstream and then calculating the total volumetric flow in each region. Then, by subtracting the near wall region from the near-air core region (and allowing for the reverse flow in the centre region) it became apparent that nearly half of the total flow was occurring over the rear of the swirl chamber, this seemed to validate the assumption of a bifurcating flow.

\subsubsection{Break-up in the outlet}

It was initially assumed that the liquid film thickness in the exit orifice remains sensibly constant, however it was seen from the high speed videos, for example Fig. 12, that in fact the continuity of the liquid film breaks down in a time dependent manner with segments of the air core actually reaching the atomizer wall. From a purely visual view the breakdown appears to be correlated with the fluctuations in the air core diameter, as discussed previously. These breakdowns of the liquid film are seen to be carried through to the spray cone, causing perforations or waves and fluctuations on the surface of the spray cone. It appears that the break-up of the liquid sheet forming the spray cone is principally caused by variations in thickness due to air core fluctuations in the atomizer allowing the formation of 'holes' which, ultimately, lead to droplet formation. In addition droplet formation could be observed to often occur in "clusters", which are traceable in their histories, back to waves on the air core. The helical waves seen in the exit orifice have the same angle as the spray cone half-angle and they thus reflect the true axial and tangential velocity components. It is also seen that the effect of the number of inlet ports is transferred throughout the atomizer and to the conical sheet.

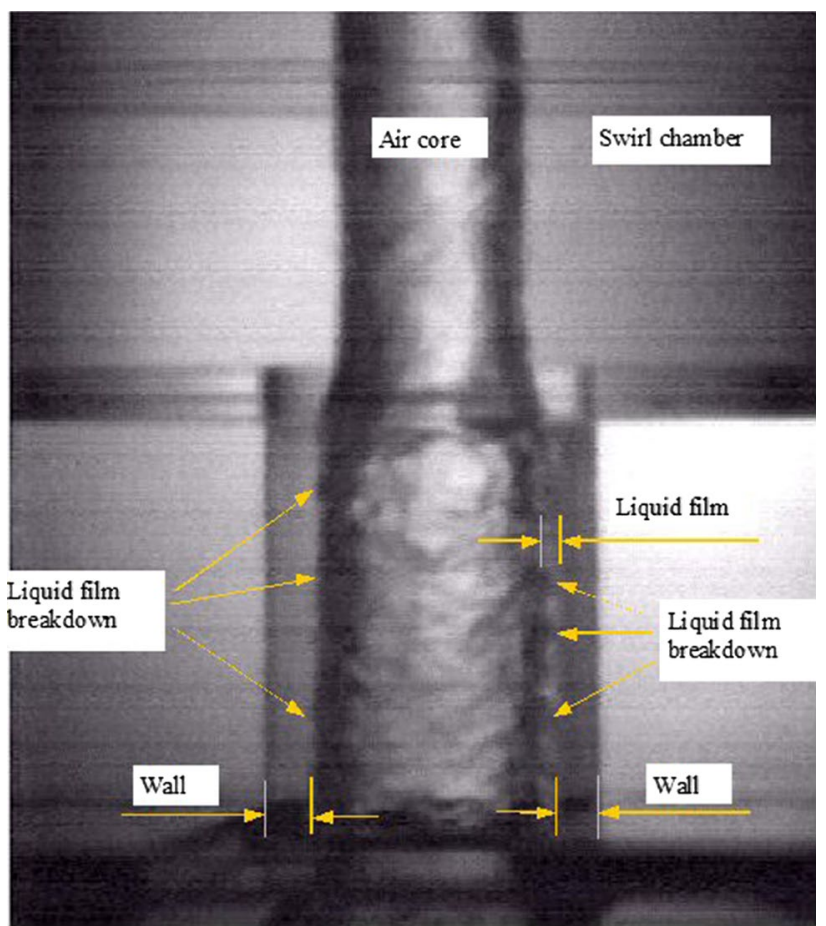

Fig. 12 Air core in the exit orifice

\section{Comparison of analytical and experimental results}

In order to calculate the theoretical wave phase velocity $\chi$ from Eq. (42) the following values of fluid properties and atomizer dimensions are used (Table 1):

This gives that the wave phase velocity $\chi$ is $2.7 \pm 0.6 \mathrm{~m} / \mathrm{s}$, to one decimal place in comparison to the experimentally recorded velocity, for this case, given in Sect. 5.2 .2 of $3.4 \pm 0.3 \mathrm{~m} / \mathrm{s}$. Thus the measured and calculated wave phase velocities are in agreement with one and other within the limits of tolerance. However, given the chaotic nature of the flow and the decreasing wave
Table 1 Dimensions and parameters of experimental model swirl atomizer flow

\begin{tabular}{lll}
\hline Outlet wall radius & $\mathrm{r}_{\mathrm{w}}=11 \mathrm{~mm}$ & $11.0 \times 10^{-3} \mathrm{~m}$ \\
Measure outlet aircore radius & $\mathrm{r}_{\mathrm{ac}}=9 \pm 0.5 \mathrm{~mm}$ & $9.0 \times 10^{-3} \mathrm{~m}$ \\
Volumetric flow rate & $\mathrm{Q}=0.4281 / \mathrm{s} 4.28 \times 10^{-4} \mathrm{~m}^{3} / \mathrm{s}$ & $4.28 \times 10^{-4} \mathrm{~m}^{3} / \mathrm{s}$ \\
Swirl chamber radius & $\mathrm{r}_{\mathrm{s}}=36.36 \mathrm{~mm}$ & $36.36 \times 10^{-3} \mathrm{~m}$ \\
Inlet height & $\mathrm{h}_{\mathrm{p}}=11 \mathrm{~mm}$ & $11.0 \times 10^{-3} \mathrm{~m}$ \\
Inlet width & $\mathrm{w}_{\mathrm{p}}=12 \mathrm{~mm}$ & $12.0 \times 10^{-3} \mathrm{~m}$ \\
Total inlet CS area & $\mathrm{A}_{\mathrm{i}}=264 \mathrm{~mm}^{2}$ & $2.64 \times 10^{-4} \mathrm{~m}^{2}$ \\
Inlet velocity $\left(\mathrm{Q} / \mathrm{A}_{\mathrm{i}}\right)$ & $\mathrm{w}_{\mathrm{i}}=1.62 \mathrm{~m} / \mathrm{s}$ & \\
Mean inlet radius $\left(\mathrm{r}_{\mathrm{s}}+\mathrm{w}_{\mathrm{p}} / 2\right)$ & $\mathrm{R}=30.36 \times 10^{-3} \mathrm{~m}$ & \\
Vorticity constant $\left(\mathrm{w}_{\mathrm{i}} \mathrm{R}\right)$ & $\mathrm{c}=4.92 \times 10^{-2} \mathrm{~m}^{2} / \mathrm{s}$ & \\
Calculated phase velocity $[\mathrm{Eq} .(25)]$ & $\chi$ & \\
\hline
\end{tabular}


phase velocity on the conical liquid sheet, as it expands, it is probably more reasonable to state that the measured and calculated velocities are in agreement with one and other to better than an order of magnitude.

\section{Conclusions}

The expression found here for the critical velocity/wave phase velocity, $\chi$, [Eqs. $(25,34,42)]$ at the throat/outlet concurs with that of Binnie and Hooking [13], by using a completely different mathematical analysis technique. Binnie and Hooking [13] mention that for the stability of flow $\mathrm{r}_{\mathrm{ac}}$ must adjust itself so that $\partial \mathrm{Q} / \partial \mathrm{r}_{\mathrm{ac}}=0$. Later Binnie [12], referring to previous work, including Unwin [22] and some of his own work, says that those writers who have employed the mass flow criterion $\left(\partial \mathrm{Q} / \partial \mathrm{r}_{\mathrm{ac}}=0\right)$ have given little justification for their analysis. He then goes on to say that for this method to be logical then the assumption $\partial \mathrm{Q} / \partial \mathrm{r}_{\mathrm{ac}}=0$ at the throat requires justification. He then asserts that $\partial \mathrm{Q} / \partial \mathrm{x}=0$ at every cross section. This is so by continuity and it is this assertion that has been worked through in the current article and on completion of the derivation it transpires that the principle of maximum flow can indeed be successfully applied to the swirl atomizer. The wave phase velocity from the first analytical treatment in the body of the atomiser gives the same expression for the velocity as the analytical treatment for the outlet flow. At this level of analysis the flow at the exit of the swirl atomizer behaves like an axi-symmetric weir. Once the optimum air core diameter is achieved it is observed that further increase in flow rate will not alter this optimum size.

High speed video and LDA measurements of the internal flow of a swirl atomizer were performed. Three distinctive types of waves were detected on the air-core liquid interface; helical striations, low frequency stationary waves and low amplitude random ripples. These waves have a range of size and degrees of orderliness.

It is believed that the helical waves are the result of the inlet flow, because the flow is not truly axisymmetric. The history of the number of inlet ports is carried through to the exit of the atomizer via helical waves. Binnie and Hookings [13] mention corrugations on the water surface of their whirlpool, probably as a residual of the two inlet flows.

Yule and Chinn [17] discovered the possibility of a second-order time-dependent flow effect of toroidal vortices within the swirl chamber of an atomizer, surrounding the air core, using Computational Fluid Dynamics. More recently, the presence of these toroidal vortices have been corroborated by Nouri-Borujerdi and Kebriaee [19], who also used numerical modelling. The video images of the stationary pulsating localised contractions/expansions of the air core diameter within the swirl chamber coincide, in size, position and frequency, with the LDA measurements of the flow velocities. The mathematical analysis carried out in the first part of this article forms a wave phase velocity that accords independently with these experimental results.

The disturbances on the air core/liquid interface caused by these waves manifest themselves in the exit region of the atomizer by the liquid film breaking down locally, with the air core itself impinging on the wall of the atomizer. The effect is, in turn, carried through to the spray cone and is manifested by low amplitude three-dimensional waves appearing on the surface of the spray cone, providing a mechanism of break-up of the liquid into droplets. Although we speak of stationary waves on the air core liquid interface within the swirl chamber it is clear that the subsequent waves, derived from these pulsations, occurring on the resultant spray cone are in transit.

Capillary waves have been found throughout the atomizer on the air-core liquid interface. Further investigation requires to be carried out to quantify and to ascertain the degree of orderliness of these waves.

Acknowledgments The authors are grateful for funding from the United Kingdom Engineering and Physical Science Research Council, Glaxo Smith Kline and Tyco Fire and Integrated Solutions Limited.

Open Access This article is distributed under the terms of the Creative Commons Attribution 4.0 International License (http://creativecommons.org/licenses/by/4.0/), which permits unrestricted use, distribution, and reproduction in any medium, provided you give appropriate credit to the original author(s) and the source, provide a link to the Creative Commons license, and indicate if changes were made.

\section{References}

1. Crapper GD, Dombrowski N, Jepson WP, Pyott GAD (1973) A note on the growth of Kelvin-Helmholtz waves on thin liquid sheets. J Fluid Mech 57(Pt 4):671-672

2. Nonnenmacher S, Piesche M (2000) Design of hollow cone pressure swirl nozzles to atomize Newtonian fluids. Chem Eng Sci 55(2000):4339-4348

3. Crapper GD (1984) Introduction to water waves. Ellis Horwood, Chichester

4. Stoker JJ (1957) Water waves. Interscience, New York

5. Taylor GI (1948) The mechanics of swirl atomizers. Seventh Int Congr Appl Mech London 2(Pt 1):280-285

6. Giffen E, Muraszew A (1953) Atomization of liquid fuels. Chapman and Hall, London

7. Bayvel L, Orzechowski Z (1993) Liquid atomization. Taylor and Francis, London

8. Nieuwkamp WC (1985) Flow analysis of a hollow cone nozzle with potential flow theory. In: Proceedings of ICLASS London IIIC/1-9

9. Yule AJ, Chinn JJ (1994) Swirl atomizer flow: classical inviscid theory revisited. In: Proceedings of ICLASS. Rouen. III-1 
10. Chinn JJ (2009) An appraisal of swirl atomizer inviscid internal flow analysis, Part 1: the principle of maximum flow for a swirl atomizer and its use in the exposition and comparison of early flow analyses. Atom Sprays J 19(3):263-282

11. Lefebvre AH (1989) Atomisation and sprays. Hemisphere, Bristol. ISBN-10:0891166033

12. Binnie AM (1949) The passage of a perfect fluid through a critical cross-section or 'throat'. In: Proceedings of the Royal Society London. A 7, vol 197

13. Binnie AM, Hookings GA (1948) Laboratory experiments on whirlpools. In: Proceedings of the Royal Society London, vol 194

14. Chinn JJ (2003) The analogy between swirl atomizer and weir flow: the principle of maximum flow. In: Proceedings of ICLASS, Sorrento Italy

15. Chinn JJ (2003) The analogy between waves on the surface of the aircore of a swirl atomizer and long, shallow water, gravity waves. In: Proceedings of ICLASS, Sorrento Italy

16. Chinn JJ, Cooper D, Yule AJ (2004) A comparison of analytically and experimentally determined wave parameters on the liquid interface of the aircore of a swirl atomizer. In: ILASS (Europe) Nottingham

17. Yule AJ, Chinn JJ (2000) The internal flow and exit conditions of pressure swirl atomizers. Atom Sprays 10(2):121-146

18. Donjat D, Estivalezes JL, Michau M, Lavergne G (2003) Phenomenological study of the pressure swirl atomizer internal flow. In: Proceedings of ICLASS, Sorrento Italy. Part 12-9

19. Nouri-Borujerdi A, Kebriaee A (2012) Numerical simulation of laminar and turbulent two-phase flow in pressure-swirl atomizers. AIAA J 50(10):2091-2101

20. Taylor GI (1950) The boundary layer in the converging nozzle of a swirl atomizer. Q J Mech Appl Math 3(Pt. 2):129-139

21. Horvay M, Leuckel W (1986) Experimental and theoretical investigation of swirl nozzles for pressure-jet atomization. Ger Chem Eng 9:276-283

22. Unwin WC (1907) A treatise on hydraulics. Black (Macmillan), New York 